\title{
*

\section{ESTUDO SOBRE OS PREÇOS DO AÇÚCAR E ALCOÓIS E A SUA INFLUÊNCIA DE LONGO PRAZO NO VOLUME DOS ESTOQUES PÚBLICOS NO BRASIL}

\section{STUDY OF THE PRICES OF SUGAR AND ALCOHOL AND ITS INFLUENCE ON LONG-TERM PUBLIC STOCKS VOLUME IN BRAZIL}

\author{
Laion Wolff* E-mail: laion london@hotmail.com \\ Adriano Mendonça Souza* E-mail: amsouza@smail.ufsm.br \\ *Universidade Federal de Santa Maria, Santa Maria, RS
}

Resumo: Neste trabalho buscou-se analisar o processo de transmissão do preço do açúcar e dos alcoóis (anidro e hidratado) e a influência do volume dos estoques públicos de açúcar no período de janeiro de 2006 a julho de 2011. Utilizaram-se na análise os testes de raiz unitária de Dickey-Fuller Aumentado (ADF) e Kwaiatkowski, Phillips, Schmidt \& Shin (KPSS) de cointegração de Johansen e o método de Auto-Regressão Vetorial com Correção de Erro (VEC). Os resultados apontam que os preços do açúcar e os preços dos alcoóis anidro e hidratado influenciam-se entre si e que o volume do estoque público de açúcar não influencia nos preços, o que já era esperado uma vez que o volume não altera o preço e sim a intervenção do governo. No geral, os choques nas variáveis que compõem o modelo são transmitidos rapidamente para a variável e são de curta duração, desaparecendo após o sexto mês.

Palavras-chave: Preço do açúcar. Preço do álcool. Agroindústria canavieira. VAR, VEC.

\begin{abstract}
This main objective of the study is to analyze transmission process between the price of sugar and prices of alcohol (anhydrous and hydrated) and the influence of the volume of public stocks of sugar, from January 2006 to July 2011. Were used in the analysis, the unit root tests of DickeyFuller (ADF), and Kwaiatkowski, Phillips, Schmidt and Shin (KPSS), of Johansen co-integration method and Vector Auto-Regression with Error Correction (VEC). The results show that sugar prices and the prices of anhydrous and hydrated alcohol influence each other, and that the volume of public stocks of sugar does not influence the prices out, what was expected, due to the volume does not change the price and but government intervention. Overall, shocks to variables in the model are transmitted quickly to the variable and are of short duration, disappearing after the sixth month.
\end{abstract}

Keywords: Price sugar. Price alcohol. Sugar-cane agroindustry. VAR. VEC. JEL Classification: C22, C51, L11.

\section{INTRODUÇÃO}

Historicamente a cana-de-açúcar é um dos principais produtos agrícolas do Brasil, sendo cultivada desde a época da colonização. Do seu processo de industrialização, obtém-se como produtos o açúcar e os alcoóis (anidro e hidratado). O álcool anidro é usado como aditivo em combustíveis, sendo composto por 99,5\% 
de álcool puro e $0,5 \%$ de água, a gasolina recebe $22 \%$ do produto. $O$ álcool hidratado possui $96 \%$ de álcool puro e $4 \%$ de água e é vendido diretamente nas bombas dos postos de gasolina.

No final da década de 1990, o Brasil passou a ser o maior produtor mundial de cana-de-açúcar. Esse grande crescimento na agroindústria canavieira foi decorrente, em grande parte, à implantação do Programa Nacional do Álcool em 1975 , o qual tinha como meta atingir a produção anual de 10,7 bilhões de litros de álcool em meados da década de 1980 e da maior inserção do Brasil no mercado internacional de açúcar.

Atualmente o Brasil é o principal exportador de açúcar no mundo e segundo Veiga (2000), pode-se creditar o crescimento das exportações brasileiras de açúcar a motivos de origem interna e externa. De origem externa, ele cita o fim do acordo bilateral entre a União das Repúblicas Socialistas Soviéticas e Cuba (URSS-Cuba), que possibilitou ao Brasil ocupar, em parte, o lugar de Cuba nesse mercado. No âmbito interno, cita as crescentes exportações oriundas do Estado de São Paulo, respaldadas pelos baixos custos de produção em relação ao de outras regiões do Brasil e do mundo.

Os preços do álcool e do açúcar sempre andaram correlacionados. Nos últimos anos, o açúcar ditou os rumos de preços do álcool, mas essa relação deverá se inverter em um futuro próximo na medida em que o combustível tem se destacado como principal produto da safra sucroalcooleira (BACCHI, 2008).

Estudos em relação aos preços de comercialização vêm sendo desenvolvidos no Brasil. Shikida (2007) por exemplo, analisou as ofertas de açúcar e álcool paranaenses a partir de uma modelagem econométrica. Como corolário, a quantidade ofertada de açúcar varia inversamente ao preço do álcool, assim como a quantidade ofertada de álcool varia inversamente ao preço do açúcar, mas esta última relação não foi significativa estatisticamente. $O$ autor concluiu que o dinamismo da agroindústria canavieira do estado do Paraná tem sido dado, mormente, pela oferta de açúcar, sendo a oferta de álcool mais residual (comparativamente à produção de açúcar).

A metodologia de vetores autorregressivos foi utilizada para identificar a relação de causalidade entre os preços do etanol e do açúcar e, de acordo com Melo (2008), existe uma relação bidirecional de causalidade entre estes preços. 
Satolo (2009) relatou o papel de choques de oferta e de demanda na evolução recente da produção de cana-de-açúcar. O modelo estimado foi um VEC modelo de Auto-Regressão Vetorial com Correção de Erro, sendo a identificação feita pelo procedimento de Bernanke- Sims. Os resultados indicam que os choques de oferta têm impacto acumulado permanente sobre a produção de cana-de-açúcar, enquanto choques de demanda apresentaram efeitos temporários. Constatou-se que os estímulos advindos da oferta foram os mais importantes para explicar as flutuações na produção de cana-de-açúcar.

Santos (2010) descreveu o comportamento dos biocombustíveis frente ao preço dos alimentos em mercados menos desenvolvidos. O comparativo entre o impacto que o preço do etanol americano causa no preço do milho no Brasil. A metodologia utilizada foi a de vetores de correção de erros (VEC) para identificar a existência de cointegração entre os mercados americano e brasileiro. Os resultados mostram que existe uma relação de cointegração entre os mercados e dão indícios de que o preço do etanol gera aumento dos preços dos alimentos no mercado brasileiro.

O objetivo deste trabalho é analisar a transmissão do preço entre o açúcar, osalcoóis anidro e hidratado e o nível de estoque público utilizando a metodologia de vetores autoregressivos e de correção de erros, de modo a verificar a influência de longo prazo entre as variáveis estudadas.

Sabe-se que, dependendo da oferta e procura, o valor do preço do açúcar e do álcool pode variar ao longo do tempo, sabendo-se também que estes preços ajudam a formar os estoques públicos, mas com uma ação de intervenção governamental para regular o preço e equilibrá-los.

Além desta breve introdução, este trabalho consta de mais três outras partes. $\mathrm{Na}$ segunda, encontram-se os dados utilizados e os procedimentos metodológicos, na terceira, descrevem-se os resultados e a discussão e, na última etapa é apresentada sua conclusão.

\section{METODOLOGIA}

Os dados utilizados nesta pesquisa compreendem o período de janeiro de 2006 a julho de 2011, referentes ao preço da saca de $50 \mathrm{~kg}$ do açúcar, preço do 
álcool anidro, preço do álcool hidratado e o volume de estoque público medido em toneladas com observação mensal. A série está disponível no site do ministério da agricultura: http://www.agricultura.gov.br/.

Os passos metodológicos adotados para a realização deste estudo estão dispostos a seguir:

Inicialmente realiza-se um teste de raízes unitárias para verificar a estabilidade das variáveis em estudo, utiliza-se o teste de Augmented Dickey-Fuller (ADF) (1979), que tem como hipótese nula a presença de raiz unitária, ou seja, não estacionariedade da série,

$\mathrm{H}_{0}: \rho=0$, a série não estacionária.

$\mathrm{H}_{1}: \rho<0$, a série é estacionária

o teste ADF é expresso pela seguinte especificação:

$$
\Delta Y_{t}=\alpha+\beta t+\eta y_{t-1}+\sum_{i=1}^{p-1} \lambda_{i} \Delta y_{t-1}+\mu \quad \text {, onde } \lambda_{i}=-\sum_{j=i+1}^{p} \rho_{j}
$$

Para a confirmação dos resultados expressos no teste anterior, utiliza-se o teste Kwaiatkowski, Phillips, Schmidt and Shin (KPSS) (1992), que possui hipóteses enunciadas de forma inversa ao teste $A D F$, sendo a hipótese nula a estacionariedade da variável a ser estudada e a hipótese alternativa como não estacionária.

$\mathrm{H}_{0}: \rho=0$, série é estacionária.

$\mathrm{H}_{1}: \rho<0$, a série é não estacionária

$O$ teste KPSS pode ser formalizado pela seguinte expressão:

$Y_{t}=\delta X_{t}+\mu_{t}$

em que $Y_{t}$ é a variável para a qual será analisada a estacionariedade, e $X_{t}$ é a variável exógena.

Esse teste tem sido utilizado pela literatura como uma contraposição aos testes em que a hipótese nula de existência de raiz unitária é testada (MADDALA e KIM, 1998).

A necessidade da determinação do grau de estacionariedade é devido à busca por se estabelecer uma relação de equilíbrio de longo prazo entre as variáveis, o que só poderá ocorrer se as variáveis possuírem o mesmo grau de integração. Logo, elas deverão ser não estacionárias em nível e após uma ou duas diferenciações deverão tornar-se estacionárias.

Revista Produção Online, Florianópolis, SC, v.13, n. 1, p. 162-179, jan./mar. 2013. 
Sendo as variáveis não estacionárias de mesma ordem, considera-se a necessidade de construção de modelos que sejam capazes de avaliar as relações existentes entre todas as variáveis sem predefinir as relações e não fazendo a distinção entre variáveis exógenas e endógenas, Sims (1980) desenvolveu os modelos de vetores autorregressivos (VAR), que devem apresentar a mesma ordem de integração.

$$
\mathbf{x}_{\mathrm{t}}=\mathbf{A}_{0}+\mathbf{A}_{\mathrm{i}} \sum_{\mathrm{i}=1}^{\mathrm{p}} \mathbf{x}_{\mathrm{t}-\mathrm{i}}+\boldsymbol{\varepsilon}_{\mathrm{t}}
$$

Sendo que $X_{t}$ é vetor das variáveis em estudo, $A_{0}$ é a matriz de interceptos $e$ $A_{i}, i=1,2, \ldots, p$ são matrizes com os termos das equações, $e_{t}$ são os erros não correlacionados, com média zero e variância constante. Ainda, $e_{t}$ é um vetor de choques não esperados nas variáveis em estudo.

Segundo Wolff (2011), na construção do modelo VAR, é importante a identificação do número de defasagens necessárias a serem incluídas no modelo. Para a seleção do número de defasagens utiliza-se o critério AIC e SBC, os quaispodem ser expressos pelas equações 4 e 5.

$$
\begin{aligned}
& A I C=N \log \left|\sum\right|+2 \theta \\
& S B C=N \log \left|\sum\right|+\theta \log (N)
\end{aligned}
$$

Sendo que $\mathrm{N}=$ número de observações utilizadas

$\log \left|\sum\right|=$ logaritmo natural do determinante da matriz de variância-covariância dos resíduos.

$\theta=$ é o número total de parâmetros em todas as equações.

Determinado o número de defasagens a ser utilizado no vetor autorregressivo, é necessário verificar se há vetores de cointegração entre as variáveis e, para essa determinação, utiliza-se a metodologia de Joahansen (EISFELD, 2007).

Deste modo, identifica-se a existência de alguma combinação linear entre as variáveis no longo prazo e conforme Engle e Granger (1987), a definição de cointegração é:

Seja $\boldsymbol{X}_{\mathrm{t}}$ um vetor $(N \times 1)$, os componentes de $\mathbf{X}_{\mathrm{t}}$ são ditos cointegrados de ordem $(d, b)$, denotado por $\mathbf{X}_{\mathrm{t}} \sim \mathrm{Cl}(d, b)$, se:

1. Todos os componentes de $\mathbf{x}_{\mathrm{t}}$ são $\mathbf{I}(d)$; 
2. Existe um vetor $\alpha \neq 0$ tal que $z_{t}=\alpha^{\prime} x_{t} \sim I(d-b), b>0$

Segundo Johansen e Juselius (1990), o número de vetores de cointegração é dado pelo teste do traço ( $\left.\lambda_{\text {Traço }}\right)$ e do máximo autovalor ( $\lambda_{\text {Max }}$ ), apresentados nas equações 6 e 7 respectivamente.

$$
\begin{gathered}
\lambda_{\text {trace }}(r)=-N \sum_{i=r+1}^{n} \ln \left(1-\lambda_{i}\right) \\
\lambda_{\max }(r, r+1)=-N \ln \left(1-\lambda_{r+1}\right)
\end{gathered}
$$

Sendo $r$ o número de cointegrações, $\mathrm{N}$ o número de observações e $\lambda$ os autovalores.

Em relação ao número de cointegrações, podem-se ter três situações: se $r=$ $\mathrm{n}$, o número de cointegrações é igual ao número de variáveis; se $r=0$, não existe nenhum vetor de cointegração e, se $0<r<n$, existem combinações lineares estacionárias em longo prazo, logo, existem $r$ vetores de cointegração (SOUZA, 2011).

Com a existência de cointegração, estima-se o vetor de correção de erros (VEC) de modo a se determinar em que período as variáveis entrarão em equilíbrio de longo prazo (HARRIS, 1995), o modelo VEC pode ser escrito conforme a equação 8.

$\Delta y_{t}=\Pi y_{t-p}+\Gamma_{1} \Delta y_{t-1}+\Gamma_{2} \Delta y_{t-2}+\ldots .+\Gamma_{p-1} \Delta y_{t-(p-1)}+\mu_{t}$

Em que $\mathrm{p}$ é o número de defasagens escolhidas no modelo VAR em uma etapa anterior. $\Pi=\beta^{\prime} \alpha$, em que $\beta$ é uma matriz $(p \times r)$, sendo em suas colunas os vetores de cointegração, e a é a matriz $(p \times r)$ contendo os coeficientes de ajustamento.

Esse modelo permite que se realizem as análises de impulso de resposta e decomposição de variância por meio de "choques" nas equações encontradas. A decomposição de variância do erro de previsão é um instrumento utilizado para descrever a dinâmica do sistema na abordagem VAR. Por esse método, torna-se possível identificar a proporção da variação total de uma variável devida a cada choque individual nas $k$ variáveis componentes do modelo. Além disso, fornece informações sobre a importância relativa de cada inovação sobre as variáveis do sistema (PINTO, 2007). 
Com o intuito de verificar a relação causal entre as variáveis, utiliza-se o teste de causalidade de Granger (1969), em que se procura determinar não apenas a direção de influência de cada variável, mas sim da existência de uma precedência temporal, tendo como pré-requisito que essa precedência venha a ser estatisticamente significativa.

Os resultados possíveis do Teste de Causalidade de Granger são:

- Causalidade unidirecional de X para Y ou de Y para X;

- Causalidade bidirecional de X para Y e de $Y$ para X;

Ausência de causalidade, quando as variáveis $\mathrm{X}$ e $\mathrm{Y}$ são independentes.

Para a realização do teste é necessário estimar as seguintes regressões de acordo com as equações 9 e 10.

$$
\begin{aligned}
& X_{t}=\sum a_{i} Y_{t-1}+\sum b_{1} X_{t-1}+U_{1 t} \\
& Y_{t}=\sum c_{i} Y_{t-1}+\sum d_{1} X_{t-1}+U_{2 t}
\end{aligned}
$$

Em que $U_{1 t}$ são os resíduos que assumimos serem não correlacionados.

A equação (9) mostra que valores correntes de $X$ estão relacionados a valores passados do próprio $\mathrm{X}$, assim como a valores defasados de $\mathrm{Y}$; a equação (10), por outro lado, mostra que os valores correntes de $Y$ estão relacionados com os valores passados de $\mathrm{X}$ e os valores defasados da variável $\mathrm{Y}$.

Definida quais as variáveis são endógenas ou exógenas, com o auxílio do teste Granger Causality/Block Exogeneity Wald, teste este baseado na estatística calculada pela equação 11, que calcula a significância conjunta de cada variável endógena defasada para cada equação do vetor autorregressivo. O teste mostra que as variáveis se ordenam das mais exógenas para as mais endógenas isto é, dos menores para os maiores valores da estatística, que pode ser representada algebricamente como:

$$
X^{2}=\sum\left[\frac{\left(f_{o}-f_{e}\right)^{2}}{f_{e}}\right]
$$

Em que $f_{0}=$ frequência observada para cada classe e $f_{e}=$ frequência esperada para aquela classe.

Para finalizar, a função impulso de resposta representa basicamente o comportamento de uma variável quando outra variável do sistema, ou ela mesma, sofre um choque (impulso) naquele determinado instante $t$, o qual se transfere para 
o período futuro em $t+1, t+2$, e assim sucessivamente. Em outras palavras, os resultados apresentados na função impulso de resposta permitem avaliar adequadamente resultados de choques em qualquer uma das variáveis do sistema. Utilizar-se-á a decomposição de Cholesky para identificar o vetor $e_{t}$.

Onde se analisa as mudanças de uma determinada variável sobre as demais variáveis do sistema, bem como constatar o tempo necessário para que tal efeito seja realizado. Dessa forma, para cada variável da equação, é submetido um choque unitário na perturbação, sendo os efeitos sobre o sistema representados graficamente. Se existem $g$ variáveis no sistema, poderá ser gerado um total de $g^{2}$ choques (BROOKS, 2003).

De acordo com Bliska (1990), uma das principais vantagens das inovações ortogonalizadas sobre as demais é serem não correlacionadas, sendo estas utilizadas no estudo. Vale lembrar que para cada ordenação das variáveis no sistema há uma decomposição diferente, sendo que a direção do efeito captado decorre da seleção arbitrária da ordem das variáveis no vetor analisado. Logo, quanto menor a covariância contemporânea, menor correlação entre os resíduos e menor será a importância da ordem selecionada. Portanto, mesmo não havendo sentido de causalidade entre duas variáveis, mesmo assim poderá haver efeito de um choque de uma sobre a outra, em função da presença de covariância entre seus respectivos erros.

Para a implementação de todas as etapas metodológicas o software estatístico utilizado foi o EViews 7.

Na próxima seção, serão apresentados os resultados encontrados por meio da aplicação da metodologia exposta anteriormente.

\section{RESULTADOS E DISCUSSÕES}

Neste item da pesquisa, são apresentados os resultados obtidos com a aplicação da metodologia exposta anteriormente e a interpretação pertinente.

Como proposto, para avaliar a estacionariedade das séries, foi realizado o teste de estacionariedade por meio do teste de ADF apresentado na Tabela 1. 
Tabela 1 - Teste da raiz unitária Augmented Dickey-Fuller (ADF)

\begin{tabular}{ccccccccc}
\hline \multirow{2}{*}{ Nível de confiança } & \multicolumn{3}{c}{ Em nível } & \multicolumn{5}{c}{ Em primeira diferença } \\
\cline { 2 - 8 } & EP & Açúcar & Anidro & Hidratado & EP & Açúcar & Anidro & Hidratado \\
\hline Valor crítico do ADF & $-1,810$ & $-1,769$ & $-2,802$ & $-1,953$ & $-7,002$ & $-5,288$ & $-9,075$ & $-4,373$ \\
$1 \%$ & $-3,519$ & $-3,520$ & $-3,519$ & $-3,519$ & $-3,519$ & $-3,520$ & $-3,519$ & $-3,519$ \\
$5 \%$ & $-2,900$ & $-2,900$ & $-2,900$ & $-2,900$ & $-2,900$ & $-2,900$ & $-2,900$ & $-2,900$ \\
$10 \%$ & $-2,587$ & $-2,587$ & $-2,587$ & $-2,587$ & $-2,587$ & $-2,587$ & $-2,587$ & $-2,587$ \\
& & & & & & & & \\
$p$-valor & 0,3725 & 0,3926 & 0,0634 & 0,3064 & $0,000^{*}$ & $0,000^{*}$ & $0,000^{*}$ & $0,000^{*}$ \\
\hline
\end{tabular}

Fonte: Elaborado pelo autor 2011, E-views 7. *Significância a 5\%

Como a hipótese $\mathrm{H}_{0}$ do teste ADF é que a série possui raiz unitária, isto é, a série é não estacionária em nível, i.e., quando não se aplica nenhuma diferença. Logo, observa-se que não se pode rejeitar a hipótese nula de que as séries possuam raiz unitária - I(1) e logo as séries são não estacionárias, pois o valor crítico do teste é menor que o valor das estatísticas calculadas para os níveis de significância estabelecidos, bem como o p-valor não foi significativo. Quando estas séries são analisadas em primeiras diferenças, não se pode rejeitar a hipótese alternativa de ausência de raiz unitária, pode-se dizer que as séries são estacionárias.

Com o objetivo de confirmar os resultados obtidos pelo teste ADF, empregouse o teste KPSS, que tem como hipótese nula a estacionariedade da série - $I(0)$, onde os resultados estão na Tabela 2.

Tabela 2 - Teste de estacionariedade Kwaiatkowski, Phillips, Schmidt and Shin (KPSS)

\begin{tabular}{cccccccccc}
\hline \multirow{2}{*}{ Nível de confiança } & \multicolumn{4}{c}{ Em nível } & \multicolumn{5}{c}{ Em primeira diferença } \\
\cline { 2 - 9 } & EP & Açúcar & Anidro & Hidratado & EP & Açúcar & Anidro & Hidratado \\
\hline Valor crítico do KPSS & 0,093 & 0,566 & 0,491 & 0,370 & 0,069 & 0,172 & 0,190 & 0,146 \\
$1 \%$ & 0,739 & 0,739 & 0,739 & 0,739 & 0,739 & 0,739 & 0,739 & 0,739 \\
$5 \%$ & 0,463 & 0,463 & 0,463 & 0,463 & 0,463 & 0,463 & 0,463 & 0,463 \\
$10 \%$ & 0,347 & 0,347 & 0,347 & 0,347 & 0,347 & 0,347 & 0,347 & 0,347 \\
& & & & & & & & \\
p-valor & 0,000 & 0,000 & 0,000 & 0,000 & 0,9674 & 0,172 & 0,863 & 0,883
\end{tabular}

Fonte: elaborado pelo autor 2011, E-views 7. 
O teste de KPSS inverte a hipótese nula (estacionariedade) contra a hipótese alternativa de existência de raiz unitária. De acordo com Kwiatkowski et al. (1992, p. 176), o teste KPSS tende a complementar o teste de raiz unitária de Dickey-Fuller (1979). Observa-se que nas séries em nível não se pode rejeitar a hipótese alternativa de não estacionariedade, enquanto que, depois de aplicada uma diferença, pode-se dizer que as séries são estacionárias. Os resultados indicam que todas as séries são estacionárias em primeira diferença, tanto pelo teste ADF como KPSS ao nível de significância de 5\%. Desta forma é possível então testar a existência de uma equação de longo prazo, pois o fato de ocorrerem séries não estacionárias em nível e com o mesmo grau de integração - I(1) é um indício da possibilidade de estimação de um modelo de correção de erros.

Logo, o primeiro procedimento da construção de um modelo de vetores autorregressivos é a escolha do número de defasagens. Os critérios utilizados para a determinação do melhor modelo foram o de AIC (Akaike Information Criterion) e o SBC (Schwarz Bayesian Criterion). Nesse processo são testadas diferentes defasagens, sendo que o menor valor dos critérios é o melhor modelo encontrado.

Tabela 3 - Valores do critério de Akaike e Schwarz para obtenção do número de defasagens a serem utilizadas para ajuste do modelo VAR

\begin{tabular}{cccc}
\hline Critérios de & \multicolumn{3}{c}{ Número de defasagens } \\
informação & 1 & 2 & 3 \\
\hline AIC & $18,56114^{*}$ & 18,66954 & 18,62159 \\
SBC & $19,42467^{*}$ & 19,87381 & 20,37569 \\
\hline
\end{tabular}

Fonte: elaborado pelo autor 2011, E-views 7.

Conforme a Tabela 3, o menor valor de AIC e SBC foi obtido ajustando o modelo com 1 defasagem para cada variável, devendo ser este o número de defasagens na estimação tanto quando for estimado o modelo VAR quanto o modelo VEC, os quais terão influência na determinação das relações de equilíbrio e nos choques utilizados.

A seguir, passa-se para o teste de Cointegração de Johansen, onde as relações de cointegração das variáveis foram definidas para o modelo VAR com uma defasagem. 
Tabela 4 - Teste da Cointegração de Johansen, teste do traço e do máximo autovalor.

\begin{tabular}{ccccccc}
\hline \multirow{2}{*}{$\begin{array}{c}\text { Número } \\
\text { de }\end{array}$} & \multicolumn{2}{c}{ Teste do Máximo Autovalor } \\
\cline { 2 - 7 } cointegração & Valor & $\begin{array}{c}\text { Estatística } \\
\text { do teste } \\
(95 \%)\end{array}$ & $p$-valor & $\begin{array}{c}\text { Valor } \\
\text { Calculado }\end{array}$ & $\begin{array}{c}\text { Estatística do } \\
\text { teste (95\%) }\end{array}$ & $p$-valor \\
\hline$r=0$ & 59,57908 & 55,24578 & 0,0198 & 31,87223 & 30,81507 & 0,0370 \\
$r \leq 1$ & 27,70684 & 35,01090 & 0,2430 & 16,51981 & 24,25202 & 0,3724 \\
$r \leq 2$ & 11,18703 & 18,39771 & 0,3733 & 7,333936 & 17,14769 & 0,6764 \\
$r \leq 3$ & 3,853097 & 3,841466 & 0,0496 & 3,853097 & 3,841466 & 0,0496 \\
\hline
\end{tabular}

Fonte: elaborado pelo autor 2011, E-views $7 .{ }^{*}$ Significativo ao nível de $5 \%$.

Os resultados apresentados na Tabela 4 indicam a estatística do máximo autovalor e do traço, nos quais se observa que se rejeita a hipótese nula de $r=0$, o qual mostra que não existe nenhum vetor de cointegração. Porém, percebe-se que existe pelo menos um vetor de cointegração, $r \leq 1$, entre as séries estudadas com nível de significância de $5 \%$ com p-valores de 0,2430 e 0,3724 , respectivamente para o teste do máximo autovalor e o teste do traço. Assim, conclui-se que há uma relação de cointegração ou relação de longo prazo entre as séries.

Constatada a presença do número de relações de cointegração entre as séries em estudo, parte-se então para a elaboração de um modelo que leve em consideração essa relação de longo prazo; para tal, utiliza-se o modelo Vetorial de Correção de Erros (VEC).

Tabela 5 - Resultados das Estimativas do Modelo de Correção de Erros (VEC) que explica a influência em longo prazo das variáveis

\begin{tabular}{ccccc}
\hline $\begin{array}{c}\text { Variável } \\
\text { explicativa }\end{array}$ & $\begin{array}{c}\text { Estatística } \\
\text { P. Açúcar }\end{array}$ & $\begin{array}{c}\text { Estatística } \\
\text { P. A. Anidro }\end{array}$ & $\begin{array}{c}\text { Estatística } \\
\text { P. A. Hidratado }\end{array}$ & $\begin{array}{c}\text { Estatística } \\
\text { V. E. Público }\end{array}$ \\
\hline P. Açúcar & 1,000 & {$[0,599]^{*}$} & {$[-0,665]^{*}$} & {$[-0,617]$} \\
P. Anidro & {$[4,897]^{*}$} & 1,000 & {$[-10,076]^{*}$} & {$[-4,895]^{*}$} \\
P. Hidratado & {$[-5,144]^{*}$} & {$[-9,529]^{*}$} & 1,000 & {$[4,626]^{*}$} \\
V. E. Público & {$[-0,775]$} & {$[-0,753]$} & {$[0,752]$} & 1,000 \\
Constante & 179,36 & 0,1844 & $-0,1305$ & $-26874,4$ \\
\hline
\end{tabular}

Fonte: Elaborado pelo autor, E-views $7 .{ }^{*}$ significativo para $1 \%>2,58$.

Percebe-se, na Tabela 5, que os coeficientes do modelo de vetores autorregressivos com correção de erros (VEC) e que as variáveis com asterisco $\left(^{*}\right)$ influenciam a variável em evidência, representada pelo coeficiente 1,000 . Portanto, o 
preço do álcool anidro e o preço do álcool hidratado apresentam uma relação de longo prazo com o preço do açúcar, e o preço do açúcar e o álcool hidratado apresentam uma relação de longo prazo com o preço do álcool anidro. Percebe-se, ainda, que o preço do açúcar e o preço do álcool anidro apresentam uma relação de longo prazo com o álcool hidratado. Já os alcoóis (hidratado e anidro) apresentam uma relação de longo prazo com o volume dos estoques públicos de açúcar.

Mesmo sendo significativo, o volume em estoques públicos tem uma variação percentual muito pequena, pois o volume presente nos estoques não influencia no preço, e sim a intervenção do governo no mercado, o que significa uma forma de regular o preço, o que vem a se confirmar com o teste de Causalidade de Granger na Tabela 6.

Tabela 6 - Teste de causalidade de Granger.

\begin{tabular}{|c|c|c|c|}
\hline Hipótese Nula & Observações & Estatística F & Probabilidade \\
\hline Açúcar não Granger Causa Estoque & & 0.58308 & 0.44796 \\
\hline Estoque não Granger Causa Açúcar & & 0.02048 & 0.88666 \\
\hline Anidro não Granger Causa Estoque & & 0.05165 & 0.82096 \\
\hline Estoque não Granger Causa Anidro & & 0.42591 & 0.51638 \\
\hline Hidratado não Granger Causa Estoque & & 0.57109 & 0.45264 \\
\hline Estoque não Granger Causa Hidratado & 66 & 1.20922 & 0.27567 \\
\hline Anidro não Granger Causa Açúcar & & 0.19699 & 0.65869 \\
\hline Açúcar não Granger Causa Anidro & & 9.34002 & 0.00329 \\
\hline Hidratado não Granger Causa Açúcar & & 0.16993 & 0.68157 \\
\hline Açúcar não Granger Causa Hidratado & & 4.30377 & 0.04212 \\
\hline Hidratado não Granger Causa Anidro & & 44.7031 & 7.0E-09 \\
\hline Anidro não Granger Causa Hidratado & & 10.9229 & 0.00157 \\
\hline
\end{tabular}

Fonte: Elaborado pelo autor, E-views 7.

Pelos resultados apresentados na Tabela 6 , pode-se identificar pelo teste de Causalidade de Granger, que o preço do açúcar causa o preço do álcool anidro e o preço do álcool hidratado. Nota-se ainda, que o preço do álcool hidratado causa o preço do álcool anidro e vice-versa, o que já era esperado pelo ajuste da oferta e procura econômica, e nenhum dos preços tem uma relação de causalidade com o volume de toneladas armazenadas em estoques públicos, corroborando a idéia de que o estoque público é instrumento de regulamentação de preço, como mostrado na discussão da Tabela 5.

Com o intuito de ordenar as variáveis de acordo com o grau de endogeneidade, foi utilizado o teste Granger Causality/Block Exogeneity, onde o valor total da estatística $X^{2}$ de cada variável mostrou o grau de importância da sua endogeneidade em relação às demais variáveis. Desse modo, a série que 
apresentou o menor valor da estatística referiu-se à variável mais exógena, e a que apresentou o maior valor, a mais endógena. O teste permitiu portanto classificar as variáveis e gerar funções de impulso de resposta, utilizou-se um critério estatisticamente consistente. Os resultados do teste permitiram ordenar as variáveis em estudo com o grau de exogeneidade.

Tabela 7 - Testes de Granger Causality/Block Exogeneity Wald

\begin{tabular}{lcccccccc}
\hline & \multicolumn{2}{c}{ Volume de Estoque } & \multicolumn{2}{c}{ Preço do Açúcar } & \multicolumn{2}{c}{ Preço do Anidro } & \multicolumn{2}{c}{ Preço do Hidratado } \\
\cline { 2 - 9 } & \multicolumn{1}{c}{$X^{2}$} & $p$-valor & $X^{2}$ & $p$-valor & $X^{2}$ & $p$-valor & $X^{2}$ & $p$-valor \\
\hline \multirow{2}{*}{ Estoque } & & & 0,005088 & 0,9431 & 0,021793 & 0,8826 & 0,187990 & 0,6646 \\
Açúcar & 4,632327 & 0,0314 & & & 0,548679 & 0,4589 & 3,952794 & 0,0468 \\
Anidro & 1,303713 & 0,2535 & 1,800903 & 0,1796 & & & 10,71553 & 0,0011 \\
Hidratado & 4,724599 & 0,0297 & 1,803103 & 0,1793 & 30,62636 & 0,0000 & & \\
\hline \multirow{2}{*}{ Total } & 6,523764 & 0,0887 & 2,006232 & 0,5711 & 44,22262 & 0,0000 & 16,17211 & 0,0010 \\
\hline
\end{tabular}

Fonte: Elaborado pelo autor, E-views 7

Como se pode notar na Tabela 7 , o preço do álcool anidro é a variável mais endógena do modelo, seguida do preço do álcool hidratado, do volume do estoque público e por último, o preço do açúcar. Para a ordenação das variáveis no modelo VAR, parte-se da variável mais exógena para a mais endógena, de forma que se tem a seguinte ordenação, representada na Tabela 8.

Tabela 8 - Ordenação da mais exógena para a mais endógena segundo o teste de Granger Causality/Block Exogeneity Wald

\begin{tabular}{llc}
\hline Mais exógeno & & $X^{2}$ \\
\hline Primeiro & Açúcar & 2,006232 \\
Segundo & Estoque & 6,523764 \\
Terceiro & Hidratado & 16,17211 \\
Quarto & Anidro & 44,22262 \\
\hline
\end{tabular}

Fonte: Elaborado pelo autor, E-views 7

O teste permitiu classificar as variáveis para gerar funções de resposta a um impulso sob um critério estatisticamente consistente.

Com os gráficos do impulso-resposta apresentado na Figura 1, é possível verificar o impacto que um choque no desvio-padrão de uma série (impulso) causa no desvio-padrão de outra série (resposta), permitindo o estudo do trajeto no tempo de cada variável endógena em relação a um choque na variável exógena.

Revista Produção Online, Florianópolis, SC, v.13, n. 1, p. 162-179, jan./mar. 2013. 
Figura 1- Gráficos de impulso-resposta.
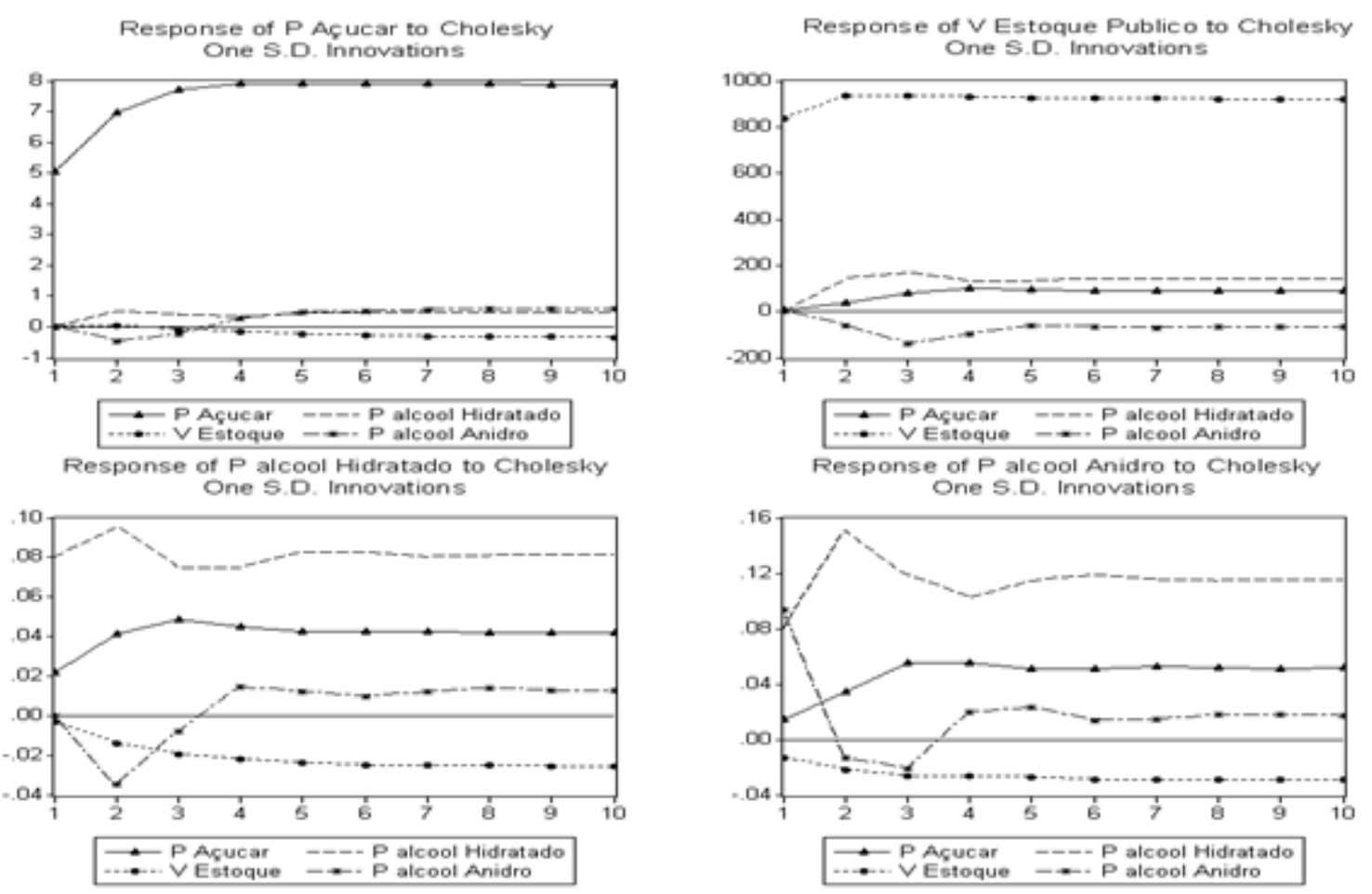

O primeiro gráfico apresenta a resposta da variação do preço do açúcar para um choque nas variáveis. Verifica-se que o preço do álcool anidro oscila negativamente, cessando o impacto a partir do quinto mês da inovação. O volume do estoque público não é afetado pela inovação, enquanto as outras duas variáveis, preço do álcool hidratado e o próprio preço do açúcar, oscilam positivamente, cessando os impactos no quarto mês.

O segundo gráfico mostra a resposta do volume do estoque público para um choque nas variáveis. Verifica-se que o choque produz uma resposta negativa para o preço do álcool anidro e positiva para as outras três variáveis, cessando o efeito a partir do quarto mês após a inovação.

O terceiro gráfico mostra a resposta do preço do álcool hidratado para um choque nas variáveis. Verifica-se que o choque produz uma resposta inicialmente positiva para o preço do açúcar e o próprio preço; e inicialmente negativa para o preço do álcool anidro e volume de estoque público, cessando o efeito a partir do quarto mês após a inovação. 
O quarto gráfico mostra a resposta do preço do álcool anidro para um choque nas variáveis. Verifica-se que o choque produz uma resposta positiva para o preço do açúcar e o preço do álcool hidratado assim como uma resposta negativa para o volume do estoque público e preço do álcool anidro, cessando o efeito a partir do quarto mês após a inovação.

Por meio dos impulsos de respostas é possível observar que qualquer informação nova ou alterações realizadas nas inovações das variáveis pode ser transmitida para as demais, causando oscilações por um curto espaço de tempo e, entrando em equilíbrio após 4 ou 6 meses no máximo. Também é importante salientar que estes choques foram aplicados utilizando um impacto em termos de 1 desvio-padrão.

\section{CONSIDERAÇÕES FINAIS}

Nesta pesquisa buscou-se determinar as relações de transmissão de preço do açúcar entre o preço dos alcoóis (anidro e hidratado). Além disso, procurou-se verificar a influência do volume de toneladas de açúcar armazenadas em estoques públicos sobre esses preços. Avaliado o pressuposto de estacionariedade, em que as séries se mostraram não estacionárias em nível, foi possível determinar uma relação de equilíbrio em longo prazo por meio da modelagem VEC, onde se identificou a existência de pelo menos um vetor de cointegração. Dessa forma, analisaram-se as relações de causalidade entre as variáveis por meio do teste de Causalidade de Granger, em que os preços se causam em todos os aspectos, e o volume armazenado em estoques públicos não tem nenhuma relação de causalidade com nenhum preço. Pela avaliação da função impulso-resposta, de um modo geral, sugere-se uma oscilação nos primeiros meses, até estabilizar a partir do quarto ao sexto mês após a inovação.

Dessa forma, foi possível verificar que a transmissão do preço do açúcar está ligada à variação do preço dos alcoóis (anidro e hidratado) e vice-versa. Observouse que o volume em estoques públicos, administrados pelo governo, não consegue controlar os preços das outras variáveis, pois esse fator não influencia no valor, e sim a intervenção do governo no mercado como uma forma de regular a cotação, 
não importando o quanto tem em volume por essa razão não apresentou resultados significativos.

\section{REFERÊNCIAS}

BACCHI, M. R. P. Álcool anidro paga melhor que o açúcar. Mercados \&Cotações 2008. Disponível em: <http://www.canaweb.com.br/pdf/178/\%5Cmerccot.pdf > Acesso em: 07 set. 2011.

BLISKA, F. M. de M. Formação de preços de carne bovina: uma aplicação do modelo de auto-regressão vetorial. Agricultura em São Paulo, São Paulo, v. 37, t. 3, p. 41-59, 1990.

BROOKS, C. Introductory Econometrics for Finance. Cambrige University Press, 2003.

CEPA. Centro de Sócio economia e Planejamento Agrícola. Disponível em: <http://cepa.epagri.sc.gov.br>. Acesso em: 26 de fev. 2011.

CONAB. Companhia Nacional de Abastecimento. Disponível em: $<$ http://www.conab.gov.br/OlalaCMS/uploads/arquivos/11 $0202 \quad 0217 \quad 1645$ arroz.p df> Acesso em: 12 de mar. 2011.

DICKEY, D.A.; FULLER, W.A. Distribution of the estimator for auto-regressive time series with a unit root. Journal of the American Statistical Association, v.74, p.427-31, 1979.

EISFELD, C.L.; NUÑEZ, B. H. C.; ALMEIDA, A.N. E SOUZA, V. S., Análise do poder de previsão do modelo de vetores autorregressivos (VAR) para a quantidade de madeira serrada exportada pelo estado do Paraná. In: ENCONTRO DE ECONOMIA PARANAENSE, PERSPECTIVA DE INSERÇÃO SOCIAL E EQUIDADE INTERNA, 5,. 2007. Anais... Curitiba - PR: EMBRAPA, 2007. Disponível em:

<http://www.embrapa.br/kw_storage/keyword.2007-07-19.4200885731>. Acesso em: 25 fev. 2011.

ENGLE, F. \& GRANGER, J. Co-integration and error correction: representation, estimation and testing. Econometrica, v. n.2. 251-276. 1987.

GRANGER, C.W. Investigating casual relations by econometric models and cross spectral methods. Econometrica 37, p.424-438. 1969.

HARRIS, R. I.D. Cointegration analysis in econometric modelling. London: Prentice Hall, 1995.

IRGA. Instituto Rio Grandense de Arroz. Disponível em:

<www.irga.rs.gov.br/docs/srcasca.pdf>. Acesso em:15 de mar. 2011. 
JOHANSEN, S.; JUSELIUS, K. Maximum likelihood estimation and inference on cointegration - with aplications to the demand for money. Oxford Bulletin of Economics and Statistics, v. 52, p.169-219, 1990.

KWIATKOWSKI, D., P. C. B. PHILLIPS, P. SCHMIDT; SHIN, Y. Testing the null hypothesis of stationarity against the alternative of a unit root: how sure are we that economic time series have a unit root?, Journal of Econometrics, 54, 159-178, 1992.

MADDALA, G.S.; KIM, In-Moo. Unit roots, cointegration and structural change.United Kingdom: Cambridge Univeristy Press, 1998.

MELO, A. S., MOTA, D. G.; LIMA, R. C., Uma análise da relação entre os preços dos biocombustíveis e das culturas alimentares no brasil: o caso do setor sucroalcooleiro. CONGRESSO SOUBER, 46,. 2008. Anais... Disponível em: <http://www.sober.org.br/palestra/9/668.pdf>. Acesso em: 12 de jan. 2012.

PINTO, A.C.B \& VIEIRA, F.V. Câmbio flexível e metas de inflação em países selecionados da América Latina: análise de vetores auto-regressivos (VAR). Revista Ensaios FEE. Porto Alegre, v. 29, n.1, 2008.

SANTOS, R. B. N.; AMORIM, A. L.; CORONEL, D. A.; SANTOS, F. T. P. Relações de co-integração entre preço dos biocombustíveis e alimentos: comparativo entre 0 etanol americano e a produção de milho no Brasil. . CONGRESSO SOUBER, 48,. 2010. Anais... Disponível em: <http://www.sober.org.br/palestra/15/198.pdf>. Acesso em: 12 de jan. 2012.

SATOLO, L. F. \& BACCHI, M.R.P. Dinâmica econômica das flutuações na produção de cana-de-açúcar. Economia Aplicada, v.13, n.3, p.377-397, 2009.

SHIKIDA, P. F. A., ALVES, L. R. A., SOUZA, E. C., CARVALHEIRO, E. M. Uma análise econométrica preliminar das ofertas de açúcar e álcool paranaenses. Rev. de Economia Agrícola, São Paulo, v. 54, n. 1, p. 21-32, jan./jun. 2007.

SIMS, C. Macroeconomics and reality. Econometrica, v. 48, p.1-48, 1980.

SOUZA, F. M.; SOUZA, A. M.; Menezes, R.. Analysis of equilibrium in industrial variables through error correction models. International Journal of Academic Research, v. 3, p. 359-364, 2011.

VEIGA FILHO, A. O dilema da "escolha de sofia" nas exportações de açúcar pelo Brasil. Informações Econômicas. São Paulo, v.30, n.9, p.53-59, set. 2000.

MAPA: Ministério da Agricultura, Pecuária e Abastecimento. Disponível em: <http://www.agricultura.gov.br/ministerio> Acesso: 01 set. 2011.

VIEIRA, N.R.A. \& OLIVEIRA, M.A.S. ARROZ. In: EMBRAPA. Ministério da agricultura, pecuária e abastecimento. Disponível em: < 
http://www.portalsaofrancisco.com.br/alfa/arroz/arroz-1.php >. Acesso em: 8 mar. 2011.

WOLFF, L.; SANTOS, E.; SOUZA, A. M. Influência do mercado acionário norte americano sobre o preço das principais ações brasileira. Organizações em contexto, São Bernardo do Campo, v. 7, n. 14, jul.- dez. 2011.

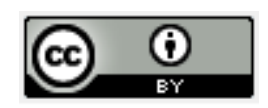

Artigo recebido em 05/10/2011 e aceito para publicação em 19/06/2012. 